\title{
NOLTA, IEICE
}

\section{FOREWORD}

\section{Special Section on Rigorous Nonlinear Analysis}

This special section is devoted to the research field of rigorous nonlinear analysis from the viewpoint of integrable system theory and verified numerical computations. They play important roles for rigorously solving linear and nonlinear problems arising in various fields of scientific research including mathematics, physics, engineering and so forth. This is a series of the special sections on verified numerical computations published in Vol. 2, No. 1 (2011), Vol. 4, No. 1 (2013), Vol. 5, No. 1 (2014), and Vol. 6, No. 3 (2015), which have attracted attention of researchers in a wide spectrum of science and engineering.

This special section includes 7 regular papers. The following variety of topics is presented: diagram of stochastic cellular automata, Hirota's bilinear approach to several nonlinear equations, graphs on the periodic discrete Toda equation, rounding errors in FFT, interval arithmetic with fixed rounding mode, accurate solutions of ill-conditioned linear systems, and the embedding constant of the Sobolev type inequality. The guest editors believe that this special section invites many readers to the frontier of this emerging research field.

We would like to express their sincere thanks to all authors for their contributions. We also thank the editorial committee members, reviewers, and all the editorial staff of NOLTA journal for their supports on publishing this special section.

\section{Daisuke Takahashi and Takeshi Ogita}

Guest Editors

\section{Editorial Committee of the Special Section}

\section{Guest Editors:}

Daisuke Takahashi (Waseda University)

Takeshi Ogita (Tokyo Woman's Christian University)

\section{Guest Associate Editors:}

Liu Xuefeng (Niigata University)

Kenichi Maruno (Waseda University)

Junta Matsukidaira (Ryukoku University)

Katsuhisa Ozaki (Shibaura Institute of Technology)

Tetsuji Tokihiro (The University of Tokyo)

Yoshitaka Watanabe (Kyushu University)

Secretaries of the Special Section:

Naoya Yamanaka (Meisei University)

Akitoshi Takayasu (University of Tsukuba)

\section{Advisory Member:}

Hiroyuki Torikai (Kyoto Sangyo University) 